PROCEEDINGS OF THE

AMERICAN MATHEMATICAL SOCIETY

Volume 127, Number 5, Pages 1339-1345

S 0002-9939(99)04621-3

Article electronically published on January 28, 1999

\title{
ASYMPTOTICS OF THE D'ALEMBERTIAN WITH POTENTIAL ON A PSEUDO-RIEMANNIAN MANIFOLD
}

\author{
THOMAS BRANSON AND GESTUR ÓLAFSSON
}

(Communicated by Palle E. T. Jorgensen)

\begin{abstract}
Let $\square$ be the Laplace-d'Alembert operator on a pseudo-Riemannian manifold $(M, g)$. We derive a series expansion for the fundamental solution $G(x, y)$ of $\square+H, H \in C^{\infty}(M)$, which behaves well under various symmetric space dualities. The qualitative properties of this expansion were used in our paper in Invent. Math. 129 (1997), 63-74, to show that the property of vanishing logarithmic term for $G(x, y)$ is preserved under these dualities.
\end{abstract}

\section{INTRODUCTION}

Let $(M, g)$ be a pseudo-Riemannian manifold of dimension $n$, with Laplaced'Alembert operator $\square$. Let $H$ be a smooth function on $M$, and consider the problem of constructing a fundamental solution for the d'Alembertian with potential $\square+H$

A classical construction of Hadamard formally develops the fundamental solution of $\square+H$ in the form

$$
G(x, y, \square+H)=\left\{\begin{array}{lc}
U(x, y) \sigma^{(2-n) / 2}+V(x, y) \log |\sigma|, & n \text { even, } \\
U(x, y)|\sigma|^{(2-n) / 2}, & n \text { odd, }
\end{array}\right.
$$

for $(x, y)$ in a neighborhood $\mathcal{O}$ of the diagonal in $M \times M$, where $\sigma=\sigma(x, y)$ is the geodesic distance-squared, and $U$ and $V$ are smooth functions on $\mathcal{O}$. Of course, the distance-squared may be negative, since the metric may be indefinite. The true fundamental solution is a version of the formal expression (1) which is regularized, either in a classical sense $[\mathrm{H}]$, or in a distributional sense $[\mathrm{C}],[\mathrm{F}]$.

The precise analytic considerations needed to produce the fundamental solution vary according to the metric signature. This subject of this paper is a classical development of the series (1) which, for certain purposes, is a valuable alternative to the Hadamard development. In particular, we used the existence of this development in an essential way in [BO]. There we proved that the vanishing of the logarithmic term $V$ is preserved under various symmetric space dualities. This, in turn, allowed us to construct many new locally symmetric spaces on which $V$ vanishes, taking the potential $H$ to be a constant multiple of the scalar curvature. The property of vanishing logarithmic term has been studied by many authors; for metrics of Lorentz

Received by the editors July 8, 1997 and, in revised form, August 6, 1997.

1991 Mathematics Subject Classification. Primary 47F05.

Research of both authors partially supported by NSF grants.

Research of the second author partially supported by a LEQSF grant.

(C)1999 American Mathematical Society 
signature, it is equivalent to Huygens' principle; for Riemannian signature, there are interpretations with consequences for classical gravitation and electrostatics. In this paper, we limit ourselves to signature-independent considerations and develop the required expansion algebraically, without treating convergence questions. In particular, all computations take place off $\{\sigma=0\}$.

Note that in this generality, the construction also produces formal asymptotic expansions of the resolvent kernels of the same operators $\square+H$; i.e., of the kernel functions for the $(\square+H-\lambda)^{-1}$, where $\lambda \in \mathbb{C}$.

In normal coordinates with origin at $y$, let $\left(x^{\alpha}\right)$ be the coordinates of the moving point $x$. The coefficients $V_{k}$ of the Taylor series for $V$,

$$
\begin{gathered}
V(x, y) \sim V_{0}(y)+\left(V_{1}\right)_{\alpha}(y) x^{\alpha}+\left(V_{2}\right)_{\alpha \beta}(y) x^{\alpha} x^{\beta}+\ldots \\
+\left(V_{k}\right)_{\alpha_{1} \ldots \alpha_{k}}(y) x^{\alpha_{1}} \ldots x^{\alpha_{k}}+\ldots,
\end{gathered}
$$

are universal local invariants of the metric $g$ and the potential $H$, valued in the symmetric $k$-tensor fields on $M$. (Here and below, the summation convention is in force.) Similar considerations hold for the function $U$. In fact, the Taylor coefficients for $U$ and $V$ may be calculated inductively and algebraically from the Taylor expansions of the metric $g$ and the potential $H$.

Choose normal coordinates for $g$ in which $y=0$ and $x=\left(x^{\alpha}\right)$. It will be convenient to introduce an artificial flat reference metric: Let $\eta$ be the standard flat metric of the same signature as $g$. Then $g$ has a normal coordinate expansion

$$
\begin{aligned}
& g_{\alpha \beta}(x)=\eta_{\alpha \beta}+g_{\alpha \beta, \gamma \delta}(y) x^{\gamma} x^{\delta}+\ldots+g_{\alpha \beta, \gamma_{1} \ldots \gamma_{k}}(y) x^{\gamma_{1}} \ldots x^{\gamma_{k}}+O\left(|x|^{k+1}\right), \\
& g^{\alpha \beta}(x)=\eta^{\alpha \beta}+g^{\alpha \beta}{ }_{, \gamma \delta}(y) x^{\gamma} x^{\delta}+\ldots+g^{\alpha \beta}{ }_{, \gamma_{1} \ldots \gamma_{k}}(y) x^{\gamma_{1}} \ldots x^{\gamma_{k}}+O\left(|x|^{k+1}\right),
\end{aligned}
$$

where the $|\cdot|$ in $O\left(|x|^{k+1}\right)$ refers to any positive definite metric. The tensors $g_{\alpha \beta, \gamma_{1} \ldots \gamma_{k}}$ and $g^{\alpha \beta}{ }_{, \gamma_{1} \ldots \gamma_{k}}$ are local invariants; that is, they are linear combinations of monomials

$$
\mathcal{C}\left(g \otimes \ldots \otimes g \otimes g^{\sharp} \otimes \ldots \otimes g^{\sharp} \otimes(\nabla \ldots \nabla R) \otimes \ldots \otimes(\nabla \ldots \nabla R)\right),
$$

where $g^{\sharp}=\left(g^{\alpha \beta}\right)$ is the inverse of the metric $g=\left(g_{\alpha \beta}\right), \nabla$ is the metric connection, $R$ is the Riemann curvature tensor of $\nabla$, and $\mathcal{C}$ is a contraction operator. As a consequence of the metric expansion (2), the normal coordinate metric determinant $\mathrm{g}$ has a similar expansion:

$$
\mathbf{g}(x)= \pm 1+\mathbf{g}_{, \alpha \beta}(y) x^{\alpha} x^{\beta}+\ldots+\mathbf{g}_{\alpha_{1} \ldots \alpha_{k}}(y) x^{\alpha_{1}} \ldots x^{\alpha_{k}}+O\left(|x|^{k+1}\right),
$$

where the tensors $\mathbf{g}_{, \alpha_{1} \ldots \alpha_{k}}$ are linear combinations of monomials of the form (3).

By Weyl's invariant theory [W], the local invariants $V_{k}$ are linear combinations of monomials of the form

$$
\begin{gathered}
\mathcal{C}\left(g \otimes \ldots \otimes g \otimes g^{\sharp} \otimes \ldots \otimes g^{\sharp} \otimes(\nabla \ldots \nabla R) \otimes \ldots \otimes(\nabla \ldots \nabla R)\right. \\
\otimes(\nabla \ldots \nabla H) \otimes \ldots \otimes(\nabla \ldots \nabla H)) .
\end{gathered}
$$

By taking note of the behavior of all terms under uniform dilation $g^{\prime}=s g, 0<$ $s \in \mathbb{R}$, it is easy to compute that each monomial (4) in $V_{k}$ (resp. $U_{j}$ ) enjoys a homogeneity property:

$$
p_{\nabla}+2\left(p_{R}+p_{H}\right)=n-2+k \quad\left(\text { resp. } p_{\nabla}+2\left(p_{R}+p_{H}\right)=j\right),
$$

where $p_{\nabla}$ (resp. $p_{R}, p_{H}$ ) is the number of $\nabla$ (resp. $R, H$ ) appearing. Note that implementation of the Ricci identities may convert occurrences of $\nabla$ into occurrences of $R$, but does not disturb the quantity $p_{\nabla}+2\left(p_{R}+p_{H}\right)$. 


\section{THE EXPANSION}

Thus far, it has not been necessary to fix sign conventions on $\square$ and $R$; we do so now. $\square_{M} f$ is the total contraction of $-g^{\sharp} \otimes \nabla \nabla f$. In a local frame $\left(X_{\alpha}\right)$,

$$
R^{\alpha}{ }_{\beta \gamma \delta} X_{\alpha}=R\left(X_{\gamma}, X_{\delta}\right) X_{\beta} .
$$

In local coordinates, the d'Alembertian is

$$
\square=-|\mathbf{g}|^{-1 / 2} \partial_{\beta}\left(g^{\alpha \beta}|\mathbf{g}|^{1 / 2} \partial_{\alpha}\right)=:-\eta^{\alpha \beta} \partial_{\alpha} \partial_{\beta}+q^{\alpha \beta}(x) \partial_{\alpha} \partial_{\beta}+b^{\alpha}(x) \partial_{\alpha} .
$$

In normal coordinates, the vanishing of the first order terms in the metric expansions gives

$$
q^{\alpha \beta}(x)=O\left(|x|^{2}\right), \quad b^{\alpha}(x)=O(|x|) .
$$

Let $D=-\eta^{\alpha \beta} \partial_{\alpha} \partial_{\beta}$. Our strategy will be to explicitly compute the effect of $D$ on homogeneous terms of the expansion (1), and to qualitatively observe the effect of $\square+H-D$. We break up a homogeneous term according to

$$
\left(V_{k}\right)_{\alpha_{1} \ldots \alpha_{k}} x^{\alpha_{1}} \ldots x^{\alpha_{k}}=V_{k}\left(x^{(k)}\right)=\sum_{\ell=0}^{[k / 2]} \sigma^{\ell} V_{k, \ell}\left(x^{(k-2 \ell)}\right),
$$

where $x^{(k)}$ is the $k$-tuple (of $n$-tuples) $(x, \ldots, x)$, and the $V_{k, \ell}$ are trace free tensors. (That is, any contraction of $V_{k, \ell}$ with $\eta^{\sharp}$ vanishes.) In representation theoretic terms, we can accomplish this by taking the symmetric tensor representation of $\mathrm{GL}(n, \mathbb{R})$ and decomposing into irreducible representations of the subgroup $\mathrm{O}(p, q)$, where $(p, q)$ is the signature of $\eta$. We similarly decompose each homogeneous term in the expansion of $U$.

For simplicity, we restrict to $\{\sigma>0\}$ for purposes of this calculation. After insertion of appropriate minus signs, it is clear that the situation on $\{\sigma<0\}$ is also described.

$D$ obeys the second order Leibniz rule $D(\varphi \psi)=\varphi D \psi+\psi D \varphi-2 \eta^{\sharp}(d \varphi, d \psi)$. But if $\Omega$ is an arbitrary trace free symmetric $p$ tensor and $\psi=\Omega\left(x^{(p)}\right)$,

$$
D \psi=0, \quad \eta^{\sharp}(d \sigma, d \psi)=2 p \psi .
$$

Furthermore, $D \sigma=-2 n$ and $\eta^{\sharp}(d \sigma, d \sigma)=4 \sigma$, so that for $s \in \mathbb{C}$,

$$
\begin{aligned}
D\left(\sigma^{s}\right) & =-2 s(n+2 s-2) \sigma^{s-1}, \\
D\left(\sigma^{s} \log \sigma\right) & =-2 \sigma^{s-1}\{s(n+2 s-2)(\log \sigma)+n+4 s-2\} .
\end{aligned}
$$

Putting all this together, we get (for $\psi$ as above)

$$
\begin{aligned}
D\left(\sigma^{s} \psi\right) & =-2 s(n+2 s+2 p-2) \sigma^{s-1} \psi, \\
D\left(\sigma^{s}(\log \sigma) \psi\right) & =-2 s(n+2 s+2 p-2) \sigma^{s-1}(\log \sigma) \psi-2(n+4 s+2 p-2) \sigma^{s-1} \psi .
\end{aligned}
$$

Thus for any $t \in \mathbb{C}$, we have

$$
\begin{aligned}
& D\left(\sigma^{\ell-t} U_{j, \ell}\left(x^{(j-2 \ell)}\right)\right)=-2(\ell-t)(n-2 \ell-2 t+2 j-2) \sigma^{\ell-t-1} U_{j, \ell}\left(x^{(j-2 \ell)}\right), \\
& D\left(\sigma^{\ell}(\log \sigma) V_{k, \ell}\left(x^{(k-2 \ell)}\right)\right) \\
& \quad=\left\{-2 \ell(n-2 \ell+2 k-2) \sigma^{\ell-1} \log \sigma-2(n+2 k-2) \sigma^{\ell-1}\right\} V_{k, \ell}\left(x^{(k-2 \ell)}\right) .
\end{aligned}
$$


In particular, setting $t=m:=(n-2) / 2$, we have

$$
\begin{aligned}
D\left(\sigma^{\ell-m} U_{j, \ell}\left(x^{(j-2 \ell)}\right)\right) & =-4(\ell-m)(j-\ell) \sigma^{\ell-m-1} U_{j, \ell}\left(x^{(j-2 \ell)}\right), \\
D\left(\sigma^{\ell}(\log \sigma) V_{k, \ell}\left(x^{(k-2 \ell)}\right)\right) & =-4 \sigma^{\ell-1}\{\ell(k-\ell+m)(\log \sigma)+k+m\} V_{k, \ell}\left(x^{(k-2 \ell)}\right) .
\end{aligned}
$$

The important qualitative point about $\square+H-D$ is (6). We have:

Lemma 1. On $\{\sigma>0\}$, for formal power series $U$ and $V$ as in (7),

$$
\begin{aligned}
(\square+ & H)\left(\sigma^{-m} U+(\log \sigma) V\right)=2 n(n-2) \sigma^{-m-1} U_{0} \\
& +\sigma^{-m-1} \sum_{j=1}^{\infty}\left\{W_{j}-\sum_{\ell=0}^{[j / 2]} 4(\ell-m)(j-\ell) \sigma^{\ell} U_{j, \ell}\left(x^{(j-2 \ell)}\right)\right\} \\
& -\sigma^{-1} \sum_{k=0}^{\infty} \sum_{\ell=0}^{[k / 2]} 4(k+m) \sigma^{\ell} V_{k, \ell}\left(x^{(k-2 \ell)}\right) \\
& +\sigma^{-1}(\log \sigma) \sum_{k=0}^{\infty}\left\{\tilde{W}_{k}-\sum_{\ell=0}^{[k / 2]} 4 \ell(k-\ell+m) \sigma^{\ell} V_{k, \ell}\left(x^{(k-2 \ell)}\right)\right\}
\end{aligned}
$$

where $W_{j}$ (resp. $\tilde{W}_{k}$ ) is a homogeneous polynomial of degree $j$ (resp. $k$ ). $W_{j, \ell}$ depends on $\left\{U_{u}\right\}$ and $\left\{V_{v}\right\}$ only through $\left\{U_{u, L} \mid u<j, L \leq \ell\right\}$ and $\left\{V_{v, L} \mid v<\right.$ $j-m, L \leq \ell\}$. $\tilde{W}_{k, \ell}$ depends on $\left\{U_{u}\right\}$ and $\left\{V_{v}\right\}$ only through $\left\{V_{v, L} \mid v<k, L \leq \ell\right\}$.

Theorem 2. If $n$ is odd, given a constant $U_{0}$, the formula of Lemma 1, with $V=0$, inductively computes a unique formal power series solution to the equation $(\square+H)\left(U|\sigma|^{-m}\right)=0$ on $\{\sigma \neq 0\}$. The coefficients $U_{j, l}(y)$ are universal local invariants as in (4). If $n$ is even, the formula of Lemma $1(b)$ inductively computes a formal power series solution to $(\square+H)\left(U \sigma^{-m}+V \log |\sigma|\right)=0$ on $\{\sigma \neq 0\}$. This solution is unique modulo solutions without singularity at $\sigma=0$. With the side conditions $U_{j, m}=0$ for all $j$, the even-dimensional solution is unique, and all coefficients $U_{j, l}(y)$ and $V_{k, l}(y)$ are universal local invariants as in (4).

Proof. First restrict to $\{\sigma>0\}$. Proceeding inductively, we find by examining the logarithm free terms that the $U_{j, \ell}$ are uniquely determined for $n$ odd. If $n$ is even,

1. The $U_{j, \ell}$ are uniquely determined for $\ell<m$ and all $j$;

2. The $U_{j, m}$ may be prescribed arbitrarily, but the $V_{k, 0}$ are uniquely determined for all $k$.

Switching attention to the terms with a $\log \sigma$ factor, we get no additional condition on the $V_{k, 0}$, and

3. The $V_{k, \ell}$ are uniquely determined for $\ell>0$ and all $k$.

Going back to the logarithm free terms,

4. The $U_{j, \ell}$ for $\ell>m$ are uniquely determined, given our prescription of the $U_{j, m}$.

If $E$ and $F$ are two power series constructed as above, differing in the prescription of the $U_{j, m}$ (and its effects on the computation of the $U_{j, \ell}$ for $\ell>m$ ), then $E-F$ is a power series (without singularity at $\sigma=0$ ) satisfying $(\square+H)(E-F)=0$. Thus the power series construction is unique up to the addition of such harmonics. 
It is straightforward to insert signs as appropriate to extend the conclusion to $\{\sigma<0\}$. The local invariance properties follow inductively from those of the metric, through (5).

The first term on the right in Lemma 1(a) regularizes to a constant multiple of the delta function when we take account of behavior through $\{\sigma=0\}$, and compute in the sense of distributions. Thus we are computing the fundamental solution of $\square+H$ by implementing the above procedure. Since $\square+H-\lambda$ is an operator of the same type, our results also cover the resolvent kernel, i.e., the kernel function of $(\square+H-\lambda)^{-1}$.

\section{Comparison with the Hadamard expansion}

Some remarks on the relation of the above results to the Hadamard expansion are in order. In Hadamard's original treatment, $U(x, y)$ and $V(x, y)$ are expanded in series of two point functions:

$$
U(x, y) \sim \sum_{j=0}^{\infty} K_{j}(x, y) \sigma^{j}, \quad V(x, y) \sim \sum_{k=0}^{\infty} L_{k}(x, y) \sigma^{k} .
$$

The $K_{j}$ and $L_{k}$ are determined, in a neighborhood of the diagonal in $M \times M$, by recursive solution of the transport equations; these are ordinary differential equations in which the geodesic parameter is the independent variable.

With $x$ as the moving point and $y$ the fixed point, the definition of $\square$ gives the following variants of (9). Let $\mathbf{G}:=\log |\mathbf{g}|$ and $r=\sigma^{1 / 2}$, and let a prime denote $d / d r$. Then

$$
\begin{aligned}
\square \sigma^{s} & =-4 s\left(m+s+\frac{1}{4} r \mathbf{G}^{\prime}\right) \sigma^{s-1}, \\
\square\left(\sigma^{s} \log \sigma\right) & =-2 \sigma^{s-1}\left\{s\left(n+2 s-2+\frac{1}{2} r \mathbf{G}^{\prime}\right)(\log \sigma)+n+4 s-2+\frac{1}{2} r \mathbf{G}^{\prime}\right\} .
\end{aligned}
$$

This gives

$$
\begin{aligned}
(\square+H)\left(K_{j} \sigma^{j-m}\right)=( & \left.(\square+H) K_{j}\right) \sigma^{j-m} \\
& -4(j-m) \sigma^{j-m-1}\left(r K_{j}^{\prime}+\left\{j+\frac{1}{4} r \mathbf{G}^{\prime}\right\} K_{j}\right), \\
(\square+H)\left(L_{k} \sigma^{k} \log \sigma\right)=( & \left.(\square+H) L_{k}\right) \sigma^{k} \log \sigma \\
& -2 k \sigma^{k-1} \log \sigma\left(2 r L_{k}^{\prime}+\left\{n+2 k-2+\frac{1}{2} r \mathbf{G}^{\prime}\right\} L_{k}\right) \\
& -2 \sigma^{k-1}\left(2 r L_{k}^{\prime}+\left\{n+4 k-2+\frac{1}{2} r \mathbf{G}^{\prime}\right\} L_{k}\right) .
\end{aligned}
$$

The analysis of, e.g., $[\mathrm{C}],[\mathrm{F}]$ shows that, after suitable regularization and normalization of $U_{0}$

$$
(\square+H)\left(\sigma^{-m} U+(\log \sigma) V\right)=\delta_{y}(x)+(\text { smooth }) .
$$

We need to make sure that $(\square+H)\left(\sigma^{-m} U+(\log \sigma) V\right)$ vanishes to infinite order for $x \neq y$. The bottom $\left(\sigma^{-m-1}\right)$ coefficient gives the condition

$$
r K_{0}^{\prime}+\frac{1}{4} r \mathbf{G}^{\prime} K_{0}=0 ;
$$

this shows that up to normalization,

$$
K_{0}(x, y)=\left(\frac{\mathbf{g}(y)}{\mathbf{g}(x)}\right)^{1 / 4} .
$$


Proceeding inductively, we then get the conditions

$$
4(j-m)\left(r K_{j}^{\prime}+\left\{j+\frac{1}{4} r \mathbf{G}^{\prime}\right\} K_{j}\right)=(\square+H) K_{j-1}, \quad 0<j<m .
$$

When we reach the $\sigma^{-1}$ coefficient in $(\square+H)\left(\sigma^{-m} U+(\log \sigma) V\right)$, we get no condition on $K_{m}$, but rather

$$
2\left(2 r L_{0}^{\prime}+\left\{n-2+\frac{1}{2} \mathbf{G}^{\prime}\right\} L_{0}\right)=(\square+H) K_{m-1} .
$$

Treating the coefficients in the $\log \sigma$ series inductively, we get

$$
2 k\left(2 r L_{k}^{\prime}+\left\{n+2 k-2+\frac{1}{2} \mathbf{G}^{\prime}\right\} L_{k}\right)=(\square+H) L_{k-1}, \quad k \geq 1 .
$$

Treating the $L_{k}$ as known and prescribing $K_{m}$ arbitrarily, we then "clean up" by solving the equations

$$
\begin{aligned}
& 4 k\left(r K_{k+m}^{\prime}+\left\{k+m+\frac{1}{4} r \mathbf{G}^{\prime}\right\} K_{k+m}\right) \\
& \quad=(\square+H) K_{k+m-1}-2\left(2 r L_{k}^{\prime}+\left\{n+4 k-2+\frac{1}{2} r \mathbf{G}^{\prime}\right\} L_{k}\right) .
\end{aligned}
$$

Through this, the ambiguity in $K_{m}$ propagates to the expansion of an arbitrary harmonic summand; this is of course the expected non-uniqueness.

Several ordinary differential equations of the form $r u^{\prime}+b u=f, b(r)=b_{0}+O\left(r^{2}\right)$, appeared in the above discussion. If $b_{0}>0$, solutions of the homogeneous equation $r u^{\prime}+b u=0$ are singular at $r=0$, so a nonsingular solution of $r u^{\prime}+b u=f$, if any, will be unique. Setting $u=r^{-b_{0}} y$, we get

$$
y^{\prime}+\beta y=r^{b_{0}-1} f, \quad \beta:=r^{-1}\left(b-b_{0}\right)=O(r) .
$$

To avoid a singularity in $u$ at $r=0$, we must take $y(0)=0$, so that

$$
y(r)=\exp \left(-\int_{0}^{r} \beta(s) d s\right) \int_{0}^{r} \exp \left(\int_{0}^{s} \beta(z) d z\right) s^{b_{0}-1} f(s) d s .
$$

If $f$ is nonsingular at $r=0$ and $b_{0}$ is a positive integer, then $y(r)=O\left(r^{b_{0}}\right)$. Thus $u$ is nonsingular at $r=0$. Since $n$ is even, inspection of the process which produces the functions $K_{j}$ and $L_{k}$ shows that they are uniquely determined and nonsingular at $r=0$.

When one actually tries to calculate the local invariants in the Hadamard expansion, attention quickly turns to the Taylor expansions of the Hadamard coefficients. The $K_{j}$ and $L_{k}$ do not have trace free Taylor coefficients; thus these coefficients do not just come from the list of $U_{j, \ell}$ and $V_{k, \ell}$ produced by our power series construction. However, the Taylor coefficients $L_{k, p}$ of the $L_{k}$ can be computed from the list $V_{k, \ell}$ and vice versa; similarly for the lists $K_{j, p}$ and $U_{j, \ell}$. The computation of any given entry from one list involves only finitely many entries from the counterpart list. The separation of $V_{k}$ into the various $V_{k, \ell}$ can be carried out effectively using $(8,9)$; that is, by taking the eigenresolution of $\sigma D$ on the space of $k$ homogeneous polynomials. The ambiguity in the definition of the $U_{j, m}$ (resp. $K_{m, p}$ ) affects only the $U_{j, q}$ (resp. the $K_{q, p}$ ) for $q \geq m$, and has no effect on the logarithmic terms.

\section{REFERENCES}

[BO] T. Branson and G. Ólafsson, Helmholtz operators and symmetric space duality, Invent. Math. 129 (1997), 63-74. CMP 97:16

[C] E. Combet, Solutions Élémentaires des Dalembertians Généralisées, Mém. Sc. Math. Facs. CLX, Gauthier-Villars, Paris, 1965.

[F] F. Friedlander, The Wave Equation on a Curved Space-time, Cambridge University Press, Cambridge, 1975. MR 57:889 
[H] J. Hadamard, Lectures on Cauchy's Problem in Linear Partial Differential Equations, Dover Publications, New York, 1952. MR 14:474f

[W] H. Weyl, The Classical Groups: Their Invariants and Representations, Princeton University Press, Princeton, 1939.

Department of Mathematics, The University of Iowa, Iowa City, Iowa 52242

E-mail address: branson@math.uiowa.edu

Department of Mathematics, Louisiana State University, Baton Rouge, Louisiana 70803

E-mail address: olafsson@marais.math.1su.edu 